investigation into the sensitivity and specificity of this test is necessary. It is possible that this may lead to a simple and reliable screening method for breast cancer using a single pubic hair.

Veronica James ${ }^{\star}$, John Kearsley $\dagger$,

Tom Irving $\ddagger$, Yoshiyuki Amemiya\$,

David Cooksong

${ }^{*}$ Rex Vowels Low Angle Diffraction Laboratory,

University of New South Wales,

Sydney, New South Wales 2052, Australia

e-mail:vjs@bigpond.com

$\dagger$ Cancer Care Centre, St George Hospital,

Kogarah 2217, Australia

$\ddagger$ BioCAT, BCPS Department,

Illinois Institute of Technology,

Chicago, Illinois 60616, USA

\$Engineering Research Unit, School of Engineering,

University of Tokyo, 2-11-16 Yayoi,

Bunkyo-ku, Tokyo 113, Japan

-Australian Nuclear Science and Technology

Organisation, Private Mail Bag 1,

Menai, New South Wales 2234, Australia

1. Struewing, J. P. et al. N. Engl. J. Med. 336, 1401-1408 (1997).

2. Wilk, K. E., James, V. J. \& Amemiya, Y. Biochim. Biophys. Acta 1245, 392-396 (1995)

3. James, V. J. \& Amemiya, Y. Textile Res. J. 68, 167-170 (1998).

\section{Change-blindness as a result of 'mudsplashes'}

Change-blindness ${ }^{1,2}$ occurs when large changes are missed under natural viewing conditions because they occur simultaneously with a brief visual disruption, perhaps caused by an eye movement ${ }^{3,4}$, a flicker ${ }^{5}$, a blink $^{6}$, or a camera cut in a film sequence ${ }^{7}$. We have found that this can occur even when the disruption does not cover or obscure the changes. When a few small, high-contrast shapes are briefly spattered over a picture, like mudsplashes on a car windscreen, large changes can be made simultaneously in the scene without being noticed. This phenomenon is potentially important in driving, sur-

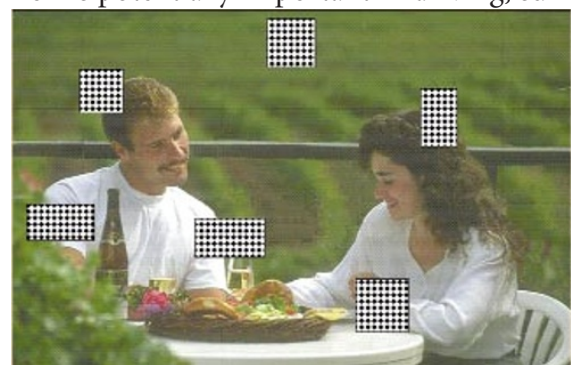

Figure 1 Mudsplashes consisted of six small blackand-white textured rectangles or ovals, dispersed over the picture so as not to cover the location of the change. The change in this example consisted of the bar in the background moving down and back up. This was judged to be a 'marginal interest' change by a panel of six judges in a previous pilot experiment. Movies of the effects, and further details can be found in Supplementary Information. veillance or navigation, as dangerous events occurring in full view can go unnoticed if they coincide with even very small, apparently innocuous, disturbances. It is also important for understanding how the brain represents the world.

We used 48 pairs of pictures, consisting of an original and a modified picture (each displayed for $3 \mathrm{~s}$ ), each pair presented cyclically with an 80 -ms duration 'mudsplash' (Fig. 1) superimposed at the moment of the change. There was no disruption in visual continuity at the moment of the change. Ten observers were asked to press a button as soon as they identified the change, which could have been a large object or region of the picture shifting in location, changing colour, or appearing or disappearing. The change could be either a 'central interest' or a 'marginal interest' element (Fig. 1). Both types of change were equalized for size and salience and were visible under normal conditions without the mudsplash.

Central-interest changes were usually detected as soon as they occurred, whereas marginal-interest changes were seen only on their second or later occurrences. In 13-30\% of the cases, marginal-interest changes, although in full view, were not detected at all during the 40-s viewing period.

Part of the explanation for these effects is that attention-grabbing luminance transitions, caused all over the visual field by the brief visual disruptions, prevent attention being focused on the location of the change. But the question remains as to why, once the extraneous transients have subsided, comparison between the current and previous views of the scene is still impossible.

Because the mudsplashes provoke only a minor disturbance, and because they do not cover the location of the change, the change-blindness they cause cannot be due to masking, or to erasure or resetting of the information contained in an internal representation of the visual world. Rather, it seems that change-blindness occurs because the internal representation of the visual world is rather sparse and essentially contains only central-interest information. A second experiment confirmed this.

Instead of mudsplashes, a single blackand-white textured rectangle briefly covered the change location at the moment of the change. This method allowed us to draw attention to the change location without giving away the exact nature of the change. When the change was in a central-interest element, observers were generally able to tell immediately what the change was. This confirms that the masking rectangle was not somehow wiping out the internal representation, and it shows that observers had coded the content of central-interest elements. When the change concerned a marginal-interest element, observers were often unable to determine what it was, showing that observers had not coded the content of marginal-interest elements.

These results indicate that humans' internal representation of the visual field is much sparser than the subjective experience of 'seeing' suggests. Only the parts of the environment that observers attend to and encode as interesting are available for making comparisons. Similar inferences have been made for sensory visual memory ${ }^{8}$, but in these older experiments, simple, artificial stimuli were used, presented for only fractions of a second, whereas we presented natural visual scenes for several seconds and used very large changes.

If only attended parts of the environment are represented in the brain, how can we have the impression of such richness and completeness in the visual world outside us? The answer might be that the visual world acts as an external memory ${ }^{9}$. We have the impression of simultaneously seeing everything, because any portion of the visual field that awakens our interest is immediately available for scrutiny through an unconscious flick of the eye or of attention. However, those parts of the scene that are not being currently processed (and are in some sense not 'seen') nevertheless constitute a background or setting that enlivens our visual experience.

The idea of the world as an outside memory is receiving attention from scientists and philosophers interested in the problem of perceptual 'filling in ${ }^{10}$ of visual scotomas and illusory contours. Work in robotics with the concept of 'active' vision is also adopting the notion that using the outside world to represent information might be more efficient than making an internal copy.

\section{J. Kevin O’Regan*, Ronald A. Rensink†,} James J. Clark

${ }^{\star}$ Laboratoire de Psychologie Expérimentale, CNRS, Université René Descartes, 28 rue Serpente,

75006 Paris, France

e-mail:oregan@ext.jussieu.fr

$\uparrow$ Cambridge Basic Research,

Nissan Research and Development,

4 Cambridge Center, Cambridge,

Massachusetts 02142, USA

$\$$ Department of Electrical Engineering,

McGill University, Montreal,

Québec H3A 2A7, Canada

1. Simons, D. J. \& Levin, D. T. Trends Cogn. Sci. 1, 261-267 (1997)

2. Intraub, H. Trends Cogn. Sci. 1, 217-221 (1997).

3. McConkie, G. W. \& Currie, C. B. J. Exp. Psychol. Hum. Percept. Perform. 22, 563-581 (1996)

4. Hayhoe, M. M., Bensinger, D. G. \& Ballard, D. H. Vision Res. 38, 125-137 (1998).

5. Rensink, R. A., O’Regan, J. K. \& Clark, J. J. Psychol. Sci. 8, 368-373 (1997).

6. O'Regan, J. K., Deubel, H., Clark, J. J. \& Rensink, R. A. Visual Cogn. (in the press).

7. Levin, D. T. \& Simons, D. J. Psychonom. Bull. Rev. 4, 501-506 (1997).

8. Haber, R. N. Behav. Brain Sci. 6, 1-54 (1983).

9. O’Regan, J. K. Can. J. Psychol. 46, 461-488 (1992).

10. Pessoa, L., Thompson, E. \& Noë, A. Behav. Brain Sci. 21, 723-802 (1998).

Supplementary information is available on Nature's World-Wide Web site (http://www.nature.com) or as paper copy from the London editorial office of Nature. 